NUCLEIC ACIDS

Nucleotide Rigidity

by our Biomolecular Structure Correspondent

THE importance and interest of the crystal structure of the dinucleoside uridylyl 3',5'-adenosine phosphate (UpA) was noted in these columns when Sussman et al. gave their preliminary account of the analysis (Nature New Biology, 233, 90; 1971). Since then an independent determination of that structure has been reported by Sundaralingam and his co-workers (Science, 174, 1020; 1971), along with detailed accounts of the analysis by both groups of workers (Sussman et al., J. Mol. Biol., 66, 403 ; 1972; and Rubin et al., Biochemistry, 11, $3112 ; 1972$ ). Apart from the degree of independence of the two analyses, which is apparently a matter of some dispute (Science, 177, 294; 1972), the two accounts have a considerable interest from the point of view of the evolution (some might say embroidery) of our ideas of nucleic acid stereochemistry.

Sussman et al. made the point that all four nucleosides in the structure (there are two dinucleosides per asymmetric unit in the crystal) have, first, the anti conformation about the sugar base link, second, the $3^{\prime}$-endo $2^{\prime}$-exo sugar pucker, and third, what might be a $\mathrm{C}-\mathrm{H}$... O hydrogen bond between the $5^{\prime} 0$ and a ring carbon of the base to which the sugar is attached. They cautiously concluded that it may well be reasonable in model building studies of polynucleotides to regard nucleosides as roughly rigid structures. Sundaralingam and colleagues note essentially the same stereochemical features but conclude somewhat more triumphantly "the concept of a rigid nucleotide unit developed here and elsewhere (Sundaralingham, in Conformations of Biological Molecules and Polymers, Bergmann, E., and Pullman, B., eds., Jerusalem, Israel Academy of Sciences and Humanities) makes a turning point in the history of nucleic acid structural research. This concept, which parallels the development of the rigid peptide unit (Pauling and Corey, 1953), is expected to have far-reaching consequences in understanding conformations of polynucleotides." This statement reminds one that even if the world does seem to be an unappreciative place, writing one's own epitaph requires some care. Perhaps with so many of the riches of molecular biology on offer to crystallographers (or to use the classic phrase "up for grabs") we should not be too sensitive to such trumpetings.

Apart from their style, however, the validity of these conclusions merits a few cautionary remarks. First, it may be that in the search for conformational certainty for polynucleotides the analogy with the peptide group was not a par- ticularly happy one. The work of Winkler and Dunitz (J. Mol. Biol., 59, 169 ; 1971) contains the conclusion that "in the construction of models of polypeptide chains or in fitting such models to electron-density maps of protein crystals, the strictly planar peptide unit thus incorporates restrictions that may be rather easily relaxed in the actual molecule." Even if it is a reasonable first assumption, however, that the amide group is planar, it should be recognized that this group with its resonance character is not at all analogous to the saturated sugar ring. It is true that in single crystal determinations of nucleotides and nucleosides two predominant types of sugar pucker are observed. Within each type, however, the standard deviation of a single torsion angle which can be used to describe the pucker is about $6^{\circ}$ corresponding to an expected range of variation in this angle of about $20^{\circ}$. Such variability about both types of sugar pucker can lead to considerable uncertainty in the expected conformation of a polynucleotide chain containing a number of sugars.

In addition to the pucker of the sugar ring, five other torsion angles are required to define the conformation of the sugar phosphate part of each nucleotide within a polynucleotide chain. Although it is true that preferred values for many of these angles are recognized (particularly from the careful surveys of Sundaralingam) it is not clear that they are anything like so well defined as the paper by Sundaralingam et al. suggests. In fact Table 9 in this paper which surveys the allowed ranges is, on close examination, not at all convincing, even when what must be a record number of errors in a published table are corrected.

The final cautionary comment on the rigid nucleotide hypothesis is a general one. What evidence we have suggests that as the crystal structures of more and more complex nucleic acid components are determined we can expect more and more surprises. The history of the observation of syn conformations about the sugar base link can be taken to point the way here, and there are already signs that stereochemical "peculiarities" occur more frequently in the structures of larger than smaller molecules-presumably as a consequence of the compromises necessary to satisfy an increasing variety of interactions. It is as well to remember that the biological structures where stereochemical information from structure determinations such as those discussed here is most relevant, such as the loops in tRNA molecules, are very often themselves very complex structures where "peculiarities" may be commonplace.

\section{NUCLEAR MAGNETIC RESONANCE Josephson Detector}

from a Correspondent

A NOVEL approach to the detection of nuclear magnetic resonance has recently been developed by Day (Phys. Rev. Lett. $29,540 ; 1972)$; the resonant radio frequency (r.f.) excitation is observed by means of a Josephson junction magnetometer (Zimmerman and Silver, Phys. Rev., 141, 367; 1966) and this new technique will allow broad lines to be measured with considerably lower r.f. field levels than previously. For example, Day has measured the magnetization of the nuclei in LiF at room temperature in a field of $7.47 \mathrm{k}$ oersted using $40 \mathrm{~dB}$ less power than is required

\title{
Difficulties in Telling QSOs from Blue Stars
}

Since the discovery of QSOs, observational astronomers have had the problem of picking these blue, star-like objects out of sky survey plates which include many genuine blue stars. One of the most useful aids in resolving this difficulty has been the rule of thumb that QSOs usually show a characteristic ultraviolet excess; but this rule breaks down on occasion, and in next Monday's Nature Physical Science I. W. A. Browne and N. J. McEwan, of the University of Manchester, discuss the identification of eleven radio sources which are believed to be associated with stellar objects "which although they appear blue on the Palomar Sky Survey Prints, do not show the characteristic ultraviolet excess".

These eleven objects are part of a group of seventeen which were noted in the Parkes $2,700 \mathrm{MHz}$ survey (Wall et al., Aust. J. Phys. (Astrophys. Suppl.), 19; 1971). All seventeen radio sources are close to blue stellar objects which have no ultraviolet excess; Browne and McEwan have now shown that more accurate radio positions still result in error boxes which include the blue stellar object in eleven of the seventeen cases. Could this result from a chance superposition of radio sources and stars? The Manchester group think not, and go on to suggest that the proposal made by Bahcall and Sargent (Astrophys. J. Lett., 148, L65; 1972), that QSOs with redshift greater than 2.3 do not exhibit ultraviolet excess, is supported by the latest observations. Their conclusion is that although two colour (blue and ultraviolet) plates are useful for confirming suspected QSOs, the technique should not be used for rejecting possible identifications. This will strike a gloomy chord for those trying to unravel the secrets of the universe by making number counts of these sources. 